\title{
Trends in alcohol consumption during pregnancy in Australia, 2001-2010
}

\author{
Sarah Callinan ${ }^{1,2}$ and Jason Ferris ${ }^{3,4}$ \\ ${ }^{1}$ Centre for Alcohol Policy Research, Turning Point Alcohol and Drug Centre, 54-62 Gertrude Street, Fitzroy, 3065, Australia \\ ${ }^{2}$ Eastern Health Clinical School, Monash University, Melbourne \\ ${ }^{3}$ Institute for Social Science Research, The University of Queensland, Australia \\ ${ }^{4}$ ARC Centre for Excellence in Policing and Security, The University of Queensland, Australia
}

\section{Abstract}

Aim: The aim of the current study is to examine, using cross-sectional data, the role of maternal age, period (year of pregnancy) and cohort (year of birth) as predictors of alcohol consumption during pregnancy over a 10-year period.

Design: Four cross-sectional surveys were examined, both separately and together.

Setting: Using cross-sectional data, there does appear to be a positive relationship between maternal age and alcohol consumption during pregnancy; however, within any one survey period, it is difficult to determine if these patterns are due to period or cohort effects.

Participants: The National Drug Strategy Household Survey (NDSHS) is a large-scale survey administered to more than 20,000 respondents. Across four survey periods, 3,281 women reported being pregnant in the 12 months prior to the survey.

Measures: The section on pregnancy and alcohol in the NDSHS 2001, 2004, 2007 and 2010.

Findings: Age was a significant positive predictor of alcohol consumption during pregnancy in 2010. However, when the four data sets were combined, period appeared to be a stronger predictor, with younger groups and cohorts decreasing consumption at a faster rate over time than older groups and cohorts.

Conclusions: Although age and cohort do play a role in the likelihood of alcohol consumption among Australian women during pregnancy, period is the most important predictor, indicating that alcohol consumption among pregnant women is decreasing. Furthermore, knowledge of pregnancy results in a marked decrease in consumption, suggesting a possible focus for prevention campaigns.

Current Australian estimates of Fetal Alcohol Spectrum Disorders (FASD) range from 0.06 to 0.68 per 1,000 live births (Mutch, Peadon, Elliott, \& Bower, 2009), and estimates of the economic cost of FASD in the United States range up to US\$8.3 billion per year (Popova, Stade, Bekmuradov, Lange, \& Rehm, 2011). FASD are likely under-reported and the economic costs to society likely underestimated (Popova, Lange, Burd, \& Rehm, 2012). FASD are caused by a pregnant woman consuming excessive alcohol during pregnancy (Khalil \& O'Brien, 2010). Aside from FASD, drinking during pregnancy has also been positively linked with preterm birth (O'Leary, Nassar, Kurinczuk, \& Bower, 2009), low birth weight (Meyer-Leu, Lemola, Daeppen, Deriaz, \& Gerber, 2011) and increased risk for sudden infant death syndrome (Athanasakis, Karavasiliadou, \& Styliadis, 2011).

Monitoring the rate of alcohol consumption during pregnancy over time is an important contribution to efforts to reduce the incidence of FASD and related disorders. It is clear that a substantial proportion of Australian women do drink at least some alcohol during pregnancy. A survey on the drinking habits of pregnant women in Sydney found that over $20 \%$ had consumed alcohol in the week prior to being surveyed (Lain, Ford, Hadfield, \& Roberts, 2010). In a study where $81 \%$ of women reported drinking alcohol during pregnancy, $0.2 \%$ of the sample reported drinking more than 20 units (i.e., standard drinks of 10 grams of

Correspondence: Sarah Callinan, Centre for Alcohol Policy Research, Turning Point Alcohol and Drug Centre, 54-62 Gertrude St, Fitzroy, VIC Australia, 3065. Telephone: +61 38413 8475; E-mail: sarahc@turningpoint.org.au

Financial support: This work was supported by the Foundation for Alcohol Research and Education, an independent, charitable organisation working to prevent the harmful use of alcohol in Australia (www.fare.org.au). Jason Ferris was also supported by the Australian Research Centre of Excellence in Policing and Security (www.ceps.edu.au/home). FARE and CEPS had no input into the design, analysis or interpretation of this study or in the decision to submit this work for publication. The authors would also like to thank Therese Reitan for her valuable insight and comments on this paper.

Keywords: Alcohol, pregnancy, age 
alcohol) a week and $71 \%$ reported 0 to 5 units per week (Mullally, Cleary, Barry, Fahey, \& Murphy, 2011).

The advice of the National Health and Medical Research Council (NHMRC) Australian Alcohol Guidelines on drinking while pregnant has changed over time. While the 1992 guidelines recommended abstinence (NHMRC, 1992), the 2001 guidelines recommended drinking less than seven standard drinks in a week, and no more than two on any one day (NHMRC, 2001). The 2009 Guidelines reverted to recommending no alcohol consumption during pregnancy, a reflection of the uncertainty surrounding the dangers of even low alcohol consumption during pregnancy (NHMRC, 2009). Despite this change, nearly a quarter of surveyed women in a recent Australian phone-based study said they intended to drink when they were pregnant (Peadon et al., 2011). A study comparing cohorts of pregnant women before and after the NHMRC guideline change in 2001 found a statistically non-significant drop in abstention in pregnant women. However, a statistically significant drop in moderate to heavy consumption, from before the guideline change to after, was detected (Powers et al., 2010). Given the switch to an abstinence-based guideline on drinking during pregnancy, much of the focus is now on research with a simple drinker-abstainer dichotomy.

Although much of the FASD prevention effort involves providing information to women about the risks of drinking during pregnancy, this approach can only be effective with women who are aware they are pregnant. Research has demonstrated that younger women are more likely to stop drinking once they become aware of their pregnancy, but are also more likely to drink before they know they are pregnant (Floyd, Decoufle, \& Hungerford, 1999). There is much to be learnt by differentiating between alcohol consumption before and after knowledge of pregnancy. Although $24 \%$ of women in a large-scale Danish birthcohort study reported binge drinking during pregnancy, the percentage of women who reported binge drinking after they had become aware of their pregnancy dropped to $4 \%$ (Strandberg-Larsen, Nielsen, Nybo, Olsen, \& Grønbæk, 2008). This is unsurprising, given that research shows that the majority of women want to at least reduce, if not completely stop, their alcohol consumption during pregnancy (Nilsen, 2009; Peadon et al., 2011).

The prevalence of alcohol consumption during pregnancy has varied over time. A survey conducted on 4,837 women who gave birth in Western Australia from 1995 to 1997 (Colvin, Payne, Parsons, Kurinczuk, \& Bower, 2007) reported that almost $60 \%$ of pregnant women had consumed alcohol. Another study found that $27.6 \%$ of a cohort of 1,186 Australian women who had children in 1999-2000, when the guidelines suggested no consumption, had drunk while pregnant, compared to $37.6 \%$ in another cohort of 1,633 women surveyed in 2003-2004, when the guidelines suggested low consumption (Maguire, 2011). While variations in the drinking patterns of pregnant women co-occur with changes in drinking guidelines, it is not clear if this rise in alcohol consumption in pregnancy can be attributed to the change in guidelines.

Another factor typically cited as important in the likelihood of alcohol consumption during pregnancy is age, although there are some conflicting results. Increased likelihood of alcohol consumption during pregnancy has been negatively linked with age (Senecky et al., 2011), and positively linked with age (Bakker et al., 2011; Palma et al., 2007), while other studies have found no age-based differences (Muckle et al., 2011).

FASDs are more common in the children of older mothers (May \& Gossage, 2011); however, the role of age as a predictor of drinking during pregnancy may be confounded by both cohort effects (that is, the year a woman was born) and period effects (that is, the year of the pregnancy). In cross-sectional studies of FASD, the main effects attributable to age may be a reflection of cohort effects, because women born more recently have been exposed to different information or social pressures on drinking during pregnancy than have women born earlier. It is important to note that these cohort effects are separate from the changes based on the time of the pregnancy. These period effects may also have an impact; for example, guidelines like those released by NHRMC could be a plausible cause for a change over time in drinking levels. All three factorsage, period and cohort-are important. However, in singular cross-sectional studies, only age varies in a testable manner; as such, any concurrent changes in drinking behavior of pregnant women attributable to age, period or cohort cannot be examined.

The primary aim of the current research is to examine the rate of self-reported drinking during pregnancy in Australia between 2001 and 2010 and to investigate the roles that age, period and cohort of pregnant women play in predicting these rates. This research draws on crosssectional data from the National Drug Strategy Household Survey (NDSHS), Australia's largest ongoing populationbased survey on drug and alcohol use and associated experiences. These data allowed us to calculate the rates of drinking during pregnancy, and explore changes in these rates given the 2009 change in national guidelines regarding drinking during pregnancy. The four time points of the NDSHS used in this research allow an examination and comparison of the respective roles that maternal age, maternal year of birth (cohort), and year of pregnancy (period) have in predicting alcohol consumption in pregnant Australian women. In addition, due to changes in the questions on pregnancy and drinking introduced in the 2010 survey, a secondary aim of this research is to differentiate between drinking before and after knowledge of pregnancy for respondents in the 2010 data.

\section{Method}

\section{Data Source}

Data were taken from the NDSHS surveys in 2001 ( $N=$ 26,744), $2004(N=29,445), 2007(N=23,356)$ and 2010 
( $N=26,648)$. Surveys were collected using the drop-andcollect method in selected Australian households. The response rate for the four surveys was between 46 and 51\% (Australian Institute of Health and Welfare, 2011). The surveys asked respondents a broad range of questions on tobacco, alcohol and illicit drug use. Only females aged 14 or over were asked the questions on pregnancy. One of the survey items asked whether the respondent had been pregnant, breastfeeding, or both pregnant and breastfeeding at any time in the past 12 months. The 3,281 women who responded "yes" to any of the questions on being pregnant were then asked about their alcohol and drug consumption during pregnancy in the 12 months prior to completing the survey. Table 1 shows the number and age of the sample taken from each survey.

Table 1.

Number and age of pregnant women in each NDSHS survey included in the current study

\begin{tabular}{lrc}
\hline Year & N & Mean Age (SD) \\
\hline 2001 & 905 & $29.9(5.73)$ \\
2004 & 896 & $29.8(5.68)$ \\
2007 & 661 & $30.8(5.89)$ \\
2010 & 819 & $30.9(5.61)$ \\
Total & 3281 & $30.3(5.74)$ \\
\hline
\end{tabular}

\section{Measures}

The primary focus in this study is on the NDSHS questions on substance use in pregnancy. Female respondents were asked a number of questions about pregnancy and breastfeeding, as well as their history of tobacco, alcohol or illicit drug consumption. Respondents who stated that they had been pregnant, or pregnant and breastfeeding, at any time in the past 12 months, were asked if they had consumed alcohol, amongst other drugs, during this time.

In 2001, 2004, and 2007, respondents were asked, “At any time in the last 12 months when you were pregnant or breastfeeding, did you use any of the following?”, with tick boxes to indicate use of a substance while pregnant or pregnant and breastfeeding. In the 2010 survey, the question was the same but the response options were changed to include before knowledge of pregnancy, after knowledge of pregnancy, and when breastfeeding.

Due to possible confusion surrounding the item on being pregnant and breastfeeding at the same time, respondents who gave this answer and later went on to indicate that they were actually neither pregnant nor breastfeeding were excluded from the sample of "pregnant women" in our current analysis. Furthermore, in all analyses in our study, the 208 women who did not answer the question on drinking during pregnancy were presumed to be indicating that they were current abstainers. This decision was made because the layout of the relevant item is unclear. The option to select "none of these" (i.e., indicating that the respondent used none of the listed substances) is easily missed, as it is the 20th of the 21 response options for this question, and it is not clear that this response is required (for more information on this decision, please see Callinan \& Room, 2012). It is important to note that there were no questions addressing the number of drinks consumed while pregnant, just whether or not any alcohol was consumed. Because of this, no analysis about binge drinking or drinking volume during pregnancy could be undertaken.

\section{Analysis}

Analysis was undertaken with Stata (version 12) (StataCorp, 2011). Participants were split into four cohort groups based on year of birth (before 1970, 1970-74, 1975-79, and 1980 or later), calculated by subtracting participants' current age from the year of the survey. They were also split into five age-based groups (under 21, 21-26, 27-32, 33-38, and 39 or older). These age and cohort groups were selected in a manner that ensured that there was a good cell size for any analysis cross-referencing age, period and cohort. The outcome was drinking behavior during pregnancy $(0=$ no and $1=$ yes $)$. Logistic regression models predicting drinking behavior during pregnancy from the explanatory variables were undertaken.

\section{Results}

Because the 2010 NDSHS items on pregnancy and drinking were slightly different from those in 2001 to 2007, it was important to ensure that the most appropriate comparison between years was made. In 2001 to 2007, respondents were asked if they had consumed alcohol while pregnant. In 2010, this was split into two separate questions, one asking if they consumed alcohol while pregnant, before knowledge of the pregnancy, and one asking if they consumed alcohol while pregnant, after knowledge of pregnancy.

Figure 1 depicts the percentage of women reporting drinking during pregnancy across the four survey periods. As can be seen in Figure 1, there was a steady decrease in women's reporting of alcohol consumption from 2001 to 2007: in 2001, 44\%, in 2004, 33\% and in 2007, $25 \%$ of women reported drinking alcohol during the most recent pregnancy. The data for 2010 diverge from this pattern. In $2010,49 \%$ of women reported drinking during pregnancy before they knew they were pregnant, while, by comparison, $20 \%$ of women reported drinking during pregnancy after they knew they were pregnant. A predicted prevalence of alcohol consumption in 2010 was generated based on the responses from 2001-2007, using regression to generate a line of best fit that was then extended to 2010 . Figure 1 shows this line, along with the reported rates of consumption.

As demonstrated in Figure 1, the 2010 item on drinking after knowledge of pregnancy better fits the trend of previous years, where there is a steady decrease in drinking during pregnancy, than drinking before knowledge of pregnancy does. While the trend line actually predicts an even lower rate of consumption than was reported for the 
item on drinking after knowledge of pregnancy in 2010, this may be due to a tapering off in the rate of decrease as the rate of consumption gets closer to zero. Therefore, in this paper, all subsequent analyses examining drinking during pregnancy from 2001 to 2010 use the drinking after knowledge of pregnancy item for the 2010 data; following on from this, we have assumed that "drinking after knowledge of pregnancy" is the implicit interpretation of the question asked of respondents in the previous three surveys.

\section{Figure 1}

Rate of reported alcohol consumption during pregnancy, 2001-2010

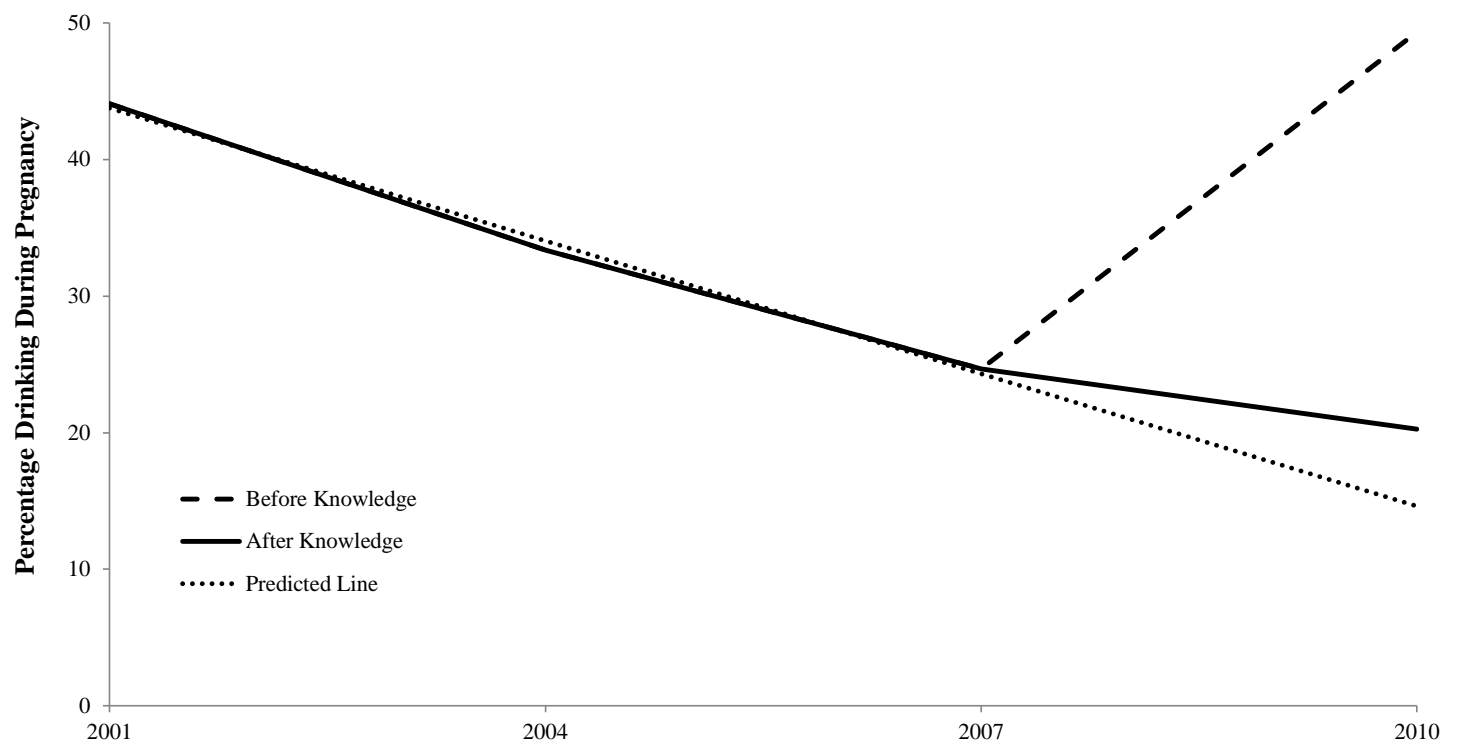

The role of maternal age as a predictor of alcohol consumption during pregnancy is examined for each of the four time periods used in this study. These four models are shown in Table 2. Age is a significant predictor of alcohol consumption during pregnancy in 2010 only; overall, there is a non-significant positive trend between alcohol consumption and age in 2004 and 2007, albeit with a dip between the youngest and the second-youngest age group.

Table 2

Logistic Regression Models Predicting Drinking during Pregnancy per Survey Year

\begin{tabular}{llllll}
\hline & & $\mathbf{2 0 0 1}$ & $\mathbf{2 0 0 4}$ & $\mathbf{2 0 0 7}$ & $\mathbf{2 0 1 0}$ \\
\hline Age & $1.14-20$ & 1 (Ref) & 1 (Ref) & 1 (Ref) & 1 (Ref) \\
& $2.21-26$ & 0.86 & 0.78 & 0.62 & 1.48 \\
& $3.27-32$ & 0.96 & 1.12 & 1.40 & 2.89 \\
& $4.33-38$ & 1.12 & 1.70 & 1.24 & $5.81^{*}$ \\
& $5 . \geq 39$ & 1.04 & 2.21 & 1.91 & $8.22^{* *}$ \\
\hline
\end{tabular}

Logistic regression models were used to investigate the role of age, period and cohort in self-reported drinking during pregnancy. The correlations between these three variables were examined to ascertain which models would be most appropriate; this is shown in Table 3. Unsurprisingly, the correlation between the cohort and age of each participant was very high; as such, models including these two variables would not be reliably interpretable (Carstensen, 2005) because entering all three variables into a model would result in an identifiability (or collinearity) problem (Rosenberg \& Anderson, 2011).

Table 3.

Correlation Matrix Displaying Year, Age and Cohort

\begin{tabular}{lcl}
\hline & Period & Cohort \\
\hline Period & 1 & \\
Cohort & $.441^{*}$ & 1 \\
Age & $.079 *$ & $-.755^{*}$ \\
\hline
\end{tabular}

$* p<.001$

Table 4 displays bivariate models demonstrating the relationship of each of these variables to alcohol consumption without controlling for any other factors or each other (Models 1-3). Model 1 highlights that age is responsible for little variance in alcohol consumption, 
although the oldest age group was more likely to consume alcohol during pregnancy than was the youngest. A Wald test did show that the relationship between the two was significant, $\chi^{2}(4)=35.20, p<.001$. In contrast, period and cohort, shown in Models 2 and 3 respectively, are both significant predictors of alcohol consumption during pregnancy. By period, the odds ratio of reporting drinking while pregnant has systematically reduced with time $\left(\chi^{2}(3)\right.$ $=104.61, p<.001)$; by cohort, those born more recently were less likely to drink $\left(\chi^{2}(3)=90.08, p<.001\right)$. This is to be expected, given the correlation between cohort and period.
To tease out the contributions of these correlated variables, multivariate models were developed. As noted, due to strong co-linearity, age and cohort could not be in the same model; as such, only two of these models were developed: one with age and period (Model 4), and one with cohort and period (Model 5). As can be seen in Table 4, where the main effects of Models 4 and 5 are shown, period remained highly significant even after age or cohort was controlled for. Cohort was still significant for all categories; however, the relationship did not appear to be as strong as it was in the bivariate model after period was accounted for.

Table 4.

Logistic Models Predicting Drinking during Pregnancy using Cohort and Year of Survey

\begin{tabular}{|c|c|c|c|c|c|c|}
\hline Variable & Category & Model 1 & Model 2 & Model 3 & Model 4 & Model 5 \\
\hline \multirow[t]{5}{*}{ Age } & $14-20$ & 1 (Ref) & & & 1 (Ref) & \\
\hline & $22-27$ & 0.76 & & & 0.80 & \\
\hline & $28-33$ & 1.09 & & & 1.16 & \\
\hline & $34-39$ & 1.38 & & & $1.56^{*}$ & \\
\hline & $\geq 40$ & $1.70^{*}$ & & & $2.02 * *$ & \\
\hline \multirow[t]{4}{*}{ Period } & 2001 & & 1 (Ref) & & 1 (Ref) & 1 (Ref) \\
\hline & 2004 & & $0.63 * * *$ & & $0.63 * * *$ & $0.69 * * *$ \\
\hline & 2007 & & $0.44^{* * *}$ & & $0.42 * * *$ & $0.52^{* * *}$ \\
\hline & 2010 & & $0.35^{* * *}$ & & $0.33^{* * *}$ & $0.47^{* * *}$ \\
\hline \multirow[t]{4}{*}{ Cohort } & $<1970$ & & & 1 (Ref) & & 1 (Ref) \\
\hline & 1970-1974 & & & $0.67 * * *$ & & $0.78^{*}$ \\
\hline & 1975-1979 & & & $0.52 * * *$ & & $0.67 * *$ \\
\hline & $\geq 1980$ & & & $0.33^{* * *}$ & & $0.47^{* * *}$ \\
\hline$R^{2}$ & & $0.009 * * *$ & $0.024^{* * *}$ & $0.027^{* * *}$ & $0.039 * * *$ & $0.036 * * *$ \\
\hline
\end{tabular}

As can be seen in the graphical representation of the interactions of Model 4 in Figure 2, the likelihood of selfreported drinking during pregnancy reduced over time for all age groups; however, the rate of this decrease varied by age, with younger women becoming increasing more likely to not drink during pregnancy over time. This interaction between age and period was significant, but only modestly $\left(\chi^{2}(12)=22.59, p=.031\right)$. While there was a slight increase in the rate of alcohol consumption from 2007 to 2010 for the two oldest age groups, this should be interpreted with caution, given the change in the wording of the question during this time.
The interaction between period and cohort in Model 5, shown in Figure 3, was similar to that of period and age. Once again, the rate of self-reported drinking during pregnancy decreased over time for all cohorts, but it decreased at a faster rate for those cohorts born more recently. This interaction between cohort and period was significant, $\chi^{2}(9)=24.56, p=.004$. There were some exceptions to this decrease in self-reported drinking rates: there was a slight increase from 2004 to 2007 in those born between 1975 and 1979. Furthermore, there was an increase in the rate of drinking from 2007 to 2010 for those born between 1970 and 1974, but given the change of wording in the drinking question, this should be interpreted with caution. 
Figure 2

Interaction between age and year of survey for alcohol consumption during pregnancy (Model 4)

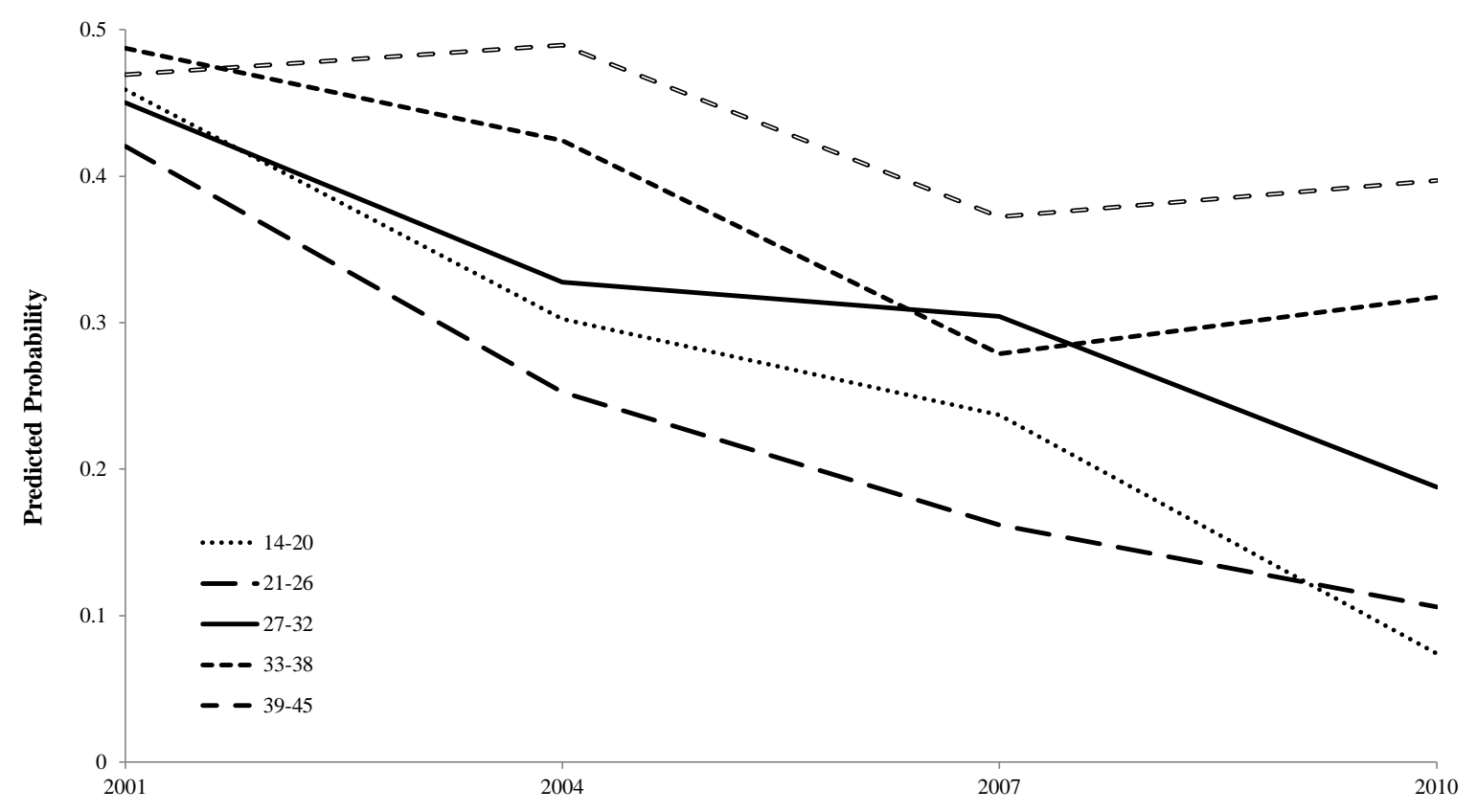

Figure 3

Interaction between cohort and year of survey for alcohol consumption during pregnancy (Model 5)

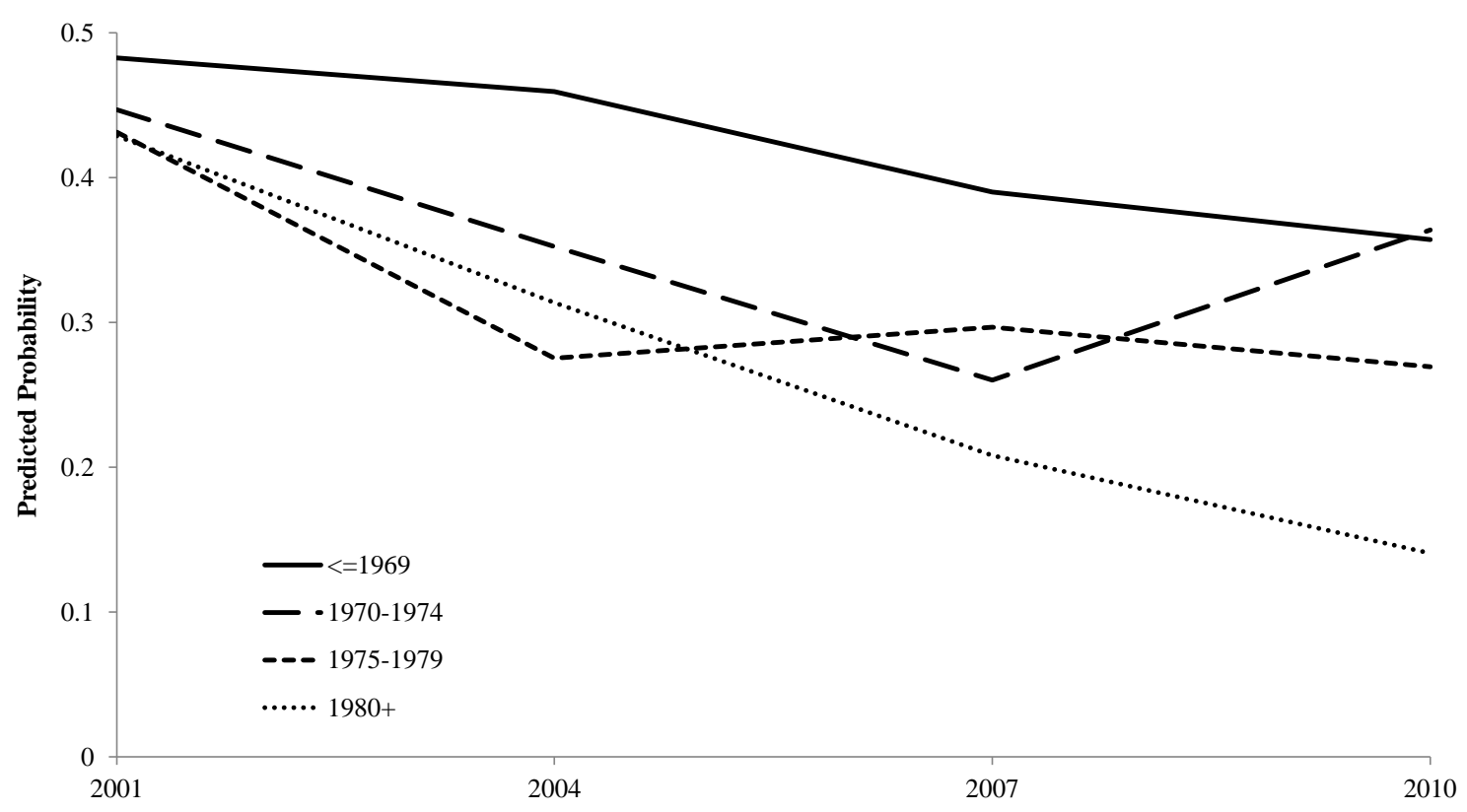




\section{Discussion}

In this study, we found a steady decrease in alcohol consumption during pregnancy in Australia in the past decade. The rate of women reporting drinking during pregnancy dropped from $44 \%$ in 2001 to $25 \%$ in 2007, with $20 \%$ drinking after knowledge of pregnancy in 2010. Out of age, period and cohort, period appears to be the strongest predictor of self-reported alcohol consumption during pregnancy, with respondents in later surveys less likely to consume alcohol than those in earlier surveys, even after age or cohort are controlled for.

By exploring the 2010 data in conjunction with the 2001 to 2007 data, we were able to get an idea of how the prevalence of drinking during pregnancy has changed during the past decade. The bivariate logistic models we used found that out of age, period and cohort, period was the strongest predictor of self-reported drinking during pregnancy, which suggests that drinking during pregnancy is decreasing in Australia. Although there is a positive correlation between age and self-reported drinking during pregnancy, this may be a reflection of a cohort effect, as well as a decrease over time in alcohol consumption during pregnancy. In support of research by May and Gossage (2011), where results from cross-sectional studies highlighted a relationship between age and alcohol-affected children, similar patterns were also found in the current study in 2010, where older pregnant women were more likely to drink. All age and cohort groups decreased alcohol consumption over time, with younger groups and more recent cohorts decreasing their alcohol consumption at a faster rate than did the older groups and cohorts. This seems to indicate that while there has been a steady decrease in alcohol consumption over the past decade, this change is not happening uniformly, and if, for instance, any of this change can be attributed to education or awareness campaigns, these campaigns are not reaching all age and cohort groups equally.

The finding that the likelihood of alcohol consumption decreased after women found out they were pregnant was not surprising, given previous research into this area that found similar results (Nilsen, 2009; Strandberg-Larsen, Nielsen, Nybo, Olsen, \& Grønbæk, 2008). More surprising was that the model that included the 2010 item on drinking after knowledge of pregnancy had better overall fit than the did model that included the 2010 item on drinking before knowledge of pregnancy. This may indicate that many women who answered the original question (which had no before or after knowledge response option) in the 2001 to 2007 surveys interpreted this question to mean drinking after knowledge of pregnancy. We assume this may be because respondents believe that drinking before knowledge of pregnancy “doesn’t count.” Alternatively, it may be that responses influenced by social desirability have increased over time, in conjunction with increasing societal pressure to remain abstinent during pregnancy.

The difficulties inherent in relying on self-reports of alcohol consumption, especially during pregnancy, need to be taken into account in this study. For instance, there is always the possibility that women are reporting less consumption over time, as compared to actually consuming less. The change in wording for the questions on drinking and pregnancy from 2007 to 2010 was a major limitation in addressing the primary research question; any change in trend from 2007 to 2010, such as the increase in consumption of those aged 33 or over, should be interpreted with caution. That said, the most anomalous results between these two time points are from increases in consumption rather than decreases, which is what would be expected, as the question on drinking after knowledge of pregnancy was used in this study. Furthermore, no information was collected on how much alcohol was consumed. Therefore, it is unclear whether high or moderate alcohol consumption is actually decreasing, or if these decreasing rates are a reflection of those who would have drunk at low levels earlier in the decade now choosing to abstain.

The initial analysis on how women are answering this question indicates that the differentiation between drinking before and after knowledge of pregnancy is valuable and should be made wherever possible in future studies. This differentiation provides the opportunity to investigate this distinction further, in conjunction with other demographic variables, so that more directly targeted programs aimed at further reducing drinking during pregnancy can be facilitated. The way this differentiation is made is also important; it is possible that drinking before knowledge of pregnancy is more socially acceptable than drinking after knowledge, despite the lack of differences in physiological ramifications between drinking before and after knowledge of pregnancy.

In conclusion, the rate of reported alcohol consumption during pregnancy in Australia is decreasing over time, and this may be more attributable to the year of pregnancy than to the age or birth cohort of the pregnant woman. Despite this, public health advocates need to be aware that the rates of drinking before knowledge of pregnancy are as high as drinking rates during pregnancy were 10 years ago. There is much work to be done in decreasing the rate of consumption before knowledge of pregnancy; also, as the rate of drinking after knowledge of pregnancy gets lower, the rate of decrease is declining. Public health campaigns should not target only those women who know they are pregnant. Rather, we recommend that there should be a campaign addressing the need to be careful of alcohol consumption during child-bearing years, as well as an investigation into the relationship between contraception methods and FASD.

\section{References}

Athanasakis, E., Karavasiliadou, S., \& Styliadis, I. (2011). The factors contributing to the risk of sudden infant death syndrome. Hippokratia, 15, 127-131.

Australian Institute of Health and Welfare (2011). 2010 National drug strategy household survey. Drug Statistic Series. Canberra: AIHW. 
Bakker, R., Steegers, E. A. P., Bilharie, A. A., Mackenbach, J. P., Hofman, A., \& Jaddoe, V. W. V. (2011). Explaining differences in birth outcomes in relation to maternal age: the Generation $\mathrm{R}$ Study. BJOG: An International Journal of Obstetrics \& Gynaecology, 118, 500-509. doi:10.111/j.14710528.2010.02823.x

Callinan, S., \& Room, R. (2012). Alcohol consumption during pregnancy: Results from the 2010 National Drug Strategy Household Survey. Canberra: Foundation for Alcohol Research and Education.

Carstensen, B. (2005). Demography and epidemiology: Age-Period-Cohort models in the computer age. Genofte, Denmark: Steno Diabetes Centre.

Colvin, L., Payne, J., Parsons, D., Kurinczuk, J. J., \& Bower, C. (2007). Alcohol consumption during pregnancy in nonindigenous West Australian women. Alcoholism: Clinical \& Experimental Research, 31, 276-284.

Floyd, R. L., Decoufle, P., \& Hungerford, D. (1999). Alcohol use prior to pregnancy recognition. American Journal of Preventative Medicine, 17, 101-107.

Khalil, A., \& O'Brien, P. (2010). Alcohol and pregnancy. Obstetrics, Gynaecology and Reproductive Medicine, 20, 311-313.

Lain, S. J., Ford, J. B., Hadfield, R. M., \& Roberts, C. L. (2010). A prevalence survey of every-day activities in pregnancy. Pregnancy and Childbirth, 10, 41. doi:1471-2393/10/41

Maguire, B. (2011). Children's pre- and perinatal health experiences. In A. I. o. F. Studies (Ed.), LSAC Annual Statistical Report 2010. Melbourne: AIFS.

May, P. A., \& Gossage, J. P. (2011). Maternal risk factors for Fetal Alcohol Spectrum Disorders: Not as simple as it might seem. Alcohol Research \& Health, 34, 15-26.

Meyer-Leu, Y., Lemola, S., Daeppen, J.-B., Deriaz, O., \& Gerber, S. (2011). Association of moderate alcohol use and binge drinking during pregnancy with neonatal health. Alcoholism Clinical and Experimental Research, 35, 1669-1677.

Muckle, G., Laflamme, D., Gagnon, J., Boucher, O., Jacobson, J., \& Jacobson, S. W. (2011). Alcohol, smoking, and drug use among Inuit women of childbearing age during pregnancy and the risk to children. Alcoholism Clinical and Experimental Research, 35, 1081-1091.

Mullally, A., Cleary, B. J., Barry, J., Fahey, T. P., \& Murphy, D. J. (2011). Prevalence, predictors and perinatal outcomes of peri-conceptional alcohol exposure-retrospective cohort study in an urban obstetric population in Ireland. BMC Pregnancy and Childbirth, 11, 27. Retrieved from http://www.biomedcentral.com/1471-2393/11/27

Mutch, R., Peadon, E. M., Elliott, E. J., \& Bower, C. (2009). Need to establish a national diagnostic capacity for foetal alcohol spectrum disorders. Journal of Paediatrics and Child Health, 45, 79-81. doi:10.1111/j.1440-1754.2009.01464.x

National Health and Medical Research Council. (1992). Is there a safe level of daily consumption of alcohol for men and women? Canberra: NHMRC.
National Health and Medical Research Council. (2001). Australian alcohol guidelines: Health risks and benefits. Canberra: NHMRC.

National Health and Medical Research Council. (2009). Australian guidelines to reduce health risks from drinking alcohol. Canberra: NHMRC.

Nilsen, P. (2009). Brief alcohol intervention to prevent drinking during pregnancy: an overview of research findings. Current Opinion in Obstetrics \& Gynaecology, 21, 496-500.

O'Leary, C. M., Nassar, N., Kurinczuk, J. J., \& Bower, C. (2009). The effect of maternal alcohol consumption on fetal growth and preterm birth. BJOG: An International Journal of Obstetrics \& Gynaecology, 116, 390-400. doi:10.1111/j.1471-0528.2008.02058.x

Palma, S., Pardo-Crespo, R., Mariscal, M., Perez-Iglesias, R., Llorca, J., \& Delgado-Rodriguez, M. (2007). Weekday but not weekend alcohol consumption before pregnancy influences alcohol cessation during pregnancy. European Journal of Public Health, 17, 394-399.

Peadon, E., Payne, J., Henley, N., D'Antoine, H., Bartu, A., O'Leary, C. M., . . . Elliott, E. J. (2011). Attitudes and behaviour predict women's intention to drink alcohol during pregnancy: the challenge for health professionals. BMC Public Health, 11, 584. Retrieved from http://www.biomedcentral.com/1471-2458/11/ 584

Popova, S., Lange, S., Burd, L., \& Rehm, J. (2012). Health care burden and cost associated with fetal alcohol syndrome in Canada. Paper presented at the 38th Annual Alcohol Epidemiology Symposium of the Kettil Bruun Society for Social \& Epidemiological Research on Alcohol, Stavanger, Norway.

Popova, S., Stade, B., Bekmuradov, D., Lange, S., \& Rehm, J. (2011). What do we know about the economic impact of Fetal Alcohol Spectrum Disorder? A systematic literature review. Alcohol and Alcoholism, 46, 490-497. doi:10.1093/alcalc/agr029

Powers, J. R., Loxton, D., Burns, L., Shakeshaft, A., Elliott, E. J., \& Dunlop, A. (2010). Assessing pregnant women's compliance with different alcohol guidelines: an 11-year prospective study. Medical Journal of Australia, 192, 690-693.

Rosenberg, P. S., \& Anderson, W. F. (2011). Age-periodcohort models in cancer surveillance research: Ready for prime time? Cancer Epidemiology Biomarkers \& Prevention, 20, 1263. doi:10.1158/1055-9965.EPI-110421

Senecky, Y., Weiss, N., Shalev, S. A., Peleg, D., Inbar, D., Chodick, G., Shalev, E., Bar-Hamburger, R. \& Shuper, A. (2011). Alcohol consumption during pregnancy among women in Israel. Journal of Population Therapeutics and Clinical Pharmacology, 18, e261e272.

StataCorp. (2011). Stata Statistical Software: Release 12. College Station, TX: StataCorp LP.

Strandberg-Larsen, K., Nielsen, N. R., Nybo, A.-M., Olsen, J., \& Grønbæk, M. (2008). Characteristics of women who binge drink before and after they become aware of their pregnancy. European Journal of Epidemiology, 23, 565-572. doi:10.1007/s10654-008-9265-z 